\title{
Cerebral venous thrombosis in a post-partum patient
}

\author{
Anushka Seneviratna ${ }^{1^{*}}$, Ramani Pallemulle ${ }^{2}$ \\ Senior Registrar in Anesthesiology ${ }^{I}$, National Hospital of Sri Lanka, Colombo, Sri Lanka, Consultant \\ Anesthetist ${ }^{2}$, Castle Street Hospital for Women, Colombo, Sri Lanka.
}

\begin{abstract}
A 39-year-old primipara delivered a healthy baby by a category 2 caesarean section as there was thick meconium and variable decelerations in the cardiotocogram (CTG). She had an uncomplicated hospital stay and was sent home on day 2. On post-partum day 18 she presented to the hospital with focal fits which had secondary generalization. The Magnetic Resonance Imaging (MRI) showed L/ anterior parietal venous haemorrhagic infarction secondary to superior sagittal sinus thrombosis. She was immediately started on low molecular weight heparin and following a repeat MRI she was discharged on warfarin with further follow up plan.
\end{abstract}

Key words: cerebral venous thrombosis; haemorrhagic venous infarction; pregnancy; post-partum

\section{Case report}

A 39-year-old mother delivered her first baby at a maternity hospital in Colombo, following a category 2 caesarean section under sub arachnoid block as there was thick meconium. She had gestational diabetes which was managed by dietary control. They were discharged on day 2 . On postpartum day 17 she started to have severe headache and some behavioural abnormalities such as forgetfulness and wandering behavior was noted. Later she had gait instability. On post-partum day 18 she had a fit at home and was brought to the local hospital. It was described as a focal fit which developed into secondary generalization. In the hospital she had a similar convulsion. Her blood pressure was normal, and the urine albumin was marginally positive. Her other routine investigations were normal. She was initiated on anti-epileptics and underwent EEG and CT scan of the brain. She was transferred to her primary maternal care hospital for MRI of brain and for further evaluation.

\footnotetext{
*Correspondence: Anushka Seneviratna E mail: anushkasenevirathna2010@gmail.com

https://orcid.org/0000 000247117412 Received: $18 / 11 / 2018$

Accepted:17/12/2018

DOI: http://doi.org/10.4038/slja.v27i1.8392
}

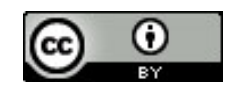

At the maternity hospital she had 3 episodes of similar convulsions. She was managed as for eclampsia and was given ICU care. The blood pressure was around $140 / 90 \mathrm{mmHg}$ with trace of albumin in urine. There were no other features of severe pre-eclampsia. There were no motor or sensory deficit and her GCS was 15/15. All her investigations were normal which included the WBC counts, platelets, liver transaminases, blood sugar and the electrolytes (serum calcium, magnesium, sodium, potassium levels). The CT brain showed probable evidence of cerebral venous thrombosis. (dense vein sign)

She was suspected to have cerebral venous thrombosis but until confirmation with urgent MRI she was managed as for eclampsia and meningoencephalitis. She was started on vancomycin, ceftriaxone and acyclovir. The MRI and MRV showed left anterior parietal venous haemorrhagic infarction secondary to superior sagittal sinus and left transverse sinus thrombosis up to the sigmoid sinus.

She was immediately started on a therapeutic dose of enoxaparin at $1 \mathrm{mg} / \mathrm{kg}$ twice daily dose. The antibiotics were omitted. She was continued on levetiracetam $1 \mathrm{~g}$ twice daily. After 4 days of ICU care she was discharged to the ward. She was monitored inward for 3 weeks for new onset neurological clinical features while continuing anticoagulants. The repeat MRI at 2 weeks showed resolving infarction in right anterior parietal lobe and partial recanalization in the superior sagittal sinus and multiple collateral formations. She was commenced on warfarin and once the therapeutic 
levels of INR was achieved, she was discharged home on warfarin. She was followed up at haematology and neurology clinics for further management.

\section{Discussion}

Cerebral venous thrombosis (CVT) is a rare disease, with an annual incidence of 3-4 per million population. ${ }^{1}$ It is more frequent during pregnancy, with an incidence of 12 per 100,000 deliveries. ${ }^{1}$ Among the sites, superior sagittal sinus thrombosis (SSST) is the commonest type of CVT.,3

CVT can present with various clinical features such as headache $(97 \%)$, seizures $(47 \%)$, neurological deficits such as paresis (43\%) and coma. ${ }^{4}$ It is commonly seen in women of 25-35 years of age and it occurs more frequently during puerperium than during pregnancy. ${ }^{5}$

Hypercoagulable state, hypovolaemia and operational deliveries are recognized as risk factors in pregnancy and puerperium for development of CVT. Further, use of oral contraceptives, prothrombotic tendencies, and infective causes related to face and head are also recognized as risk factors. ${ }^{3}$

Our patient had headache, behavioural abnormalities and gait imbalance. Later she developed convulsions on post-partum day 18 . These features combined with the focal origin of the convulsions favour the most likely diagnosis as cerebral venous thrombosis. In the postpartum period, the headache from CVT is often misdiagnosed as post dural puncture headache. In this case it was not considered as the patient presented late, on day 18. The other possible differential diagnosis such as eclampsia and meningo-encephalitis should always be considered in this setting.

We initially treated this patient as eclampsia until the definitive diagnosis was made even though she had less features to favour pre-eclampsia. There was mild neck stiffness but had no fever and the WBC and the CRP was normal. Therapeutic doses of antibiotics were started to cover meningoencephalitis as well.
Neuro imaging confirms the diagnosis of CVT. A CT scan is a useful initial examination technique which will rule out other acute cerebral disorders and it will also show venous infarcts or haemorrhages, but it's results can be entirely normal in $70 \%$ of the patients. The most sensitive examination technique and imaging modality of choice is MRI with gadolinium in combination with MR venography. MRI will provide details on both brain parenchyma and cerebral venous system (veins and sinuses). It will directly visualize the venous thrombus within the vessel. MRV will show non-visualization of vessel, flow defects and new collaterals. Therefore, both in combination are sensitive and specific enough to provide the best noninvasive method of diagnosing cerebral venous thrombosis. ${ }^{6,7}$

Treatment options for cerebral venous sinus thrombosis include anticoagulation, thrombolytic therapy and in some cases surgical thrombectomy.

The dilemma in our patient in starting systemic anti coagulation was the presence of the venous haemorrhagic infarction. We conducted a multidisciplinary team discussion involving the anaesthetist, obstetrician, haematologist and the neurologist. It was discussed that the venous haemorrahge was as a consequence of the back pressure due to significant thrombosis. The consensus decision was to start systemic anti coagulation with enoxaparin at $1 \mathrm{mg} / \mathrm{kg}$ dose while monitoring the patient inward. As the repeat MRI done at 2 weeks showed improving features she was started on warfarin. The bridging therapy was continued until she achieved the therapeutic ranges of INR.

Recognized complications of CVT are intracranial hypertension and hydrocephalus. They should be monitored for these complications. If there is a rise in the intra cranial pressure and deterioration in the patient's neurology, decompression craniotomy should be considered.

Prognosis of venous sinus thrombosis is quite variable. The outcome can result from total recovery to death, independent survival rates of approximately $80 \%$ have been stated. ${ }^{8}$ The risk of 
recurrent cerebral venous sinus thrombosis in future pregnancies and puerperium is said to be low. ${ }^{8}$

Our patient had no neurological complications and she was discharged home following successful anticoagulation with further follow up plans.

\section{Conclusion}

CVT is an uncommon diagnosis in pregnancy and puerperium and if left undiagnosed could lead to life threatening complications. Yet its management is simple with an excellent prognosis. Thus, timely intervention and correct diagnosis could save lives and prevent debilitating consequences.

\section{References}

1. Stam J. Thrombosis of cerebral veins and sinuses. New England Journal of Medicine,2005;352:17918.

https://doi.org/10.1056/NEJMra042354

PMid: 15858188

2. Sasidharan PK. Cerebral Vein Thrombosis misdiagnosed and mismanaged, Thrombosis, 2012; Article ID 210676, 11pages.

3. Ganesh Dangal G, Thapa LB. Cerebral venous sinus thrombosis presenting in pregnancy and puerperium. BMJ case reports. PMC3029151 2009.

4. de Bruin S, de Hann R, Stam J. Clinical features and prognostic factors of cerebral venous sinus thrombosis in a prospective series of 59 patients. $\mathrm{J}$ NeurolNeurosurgPsychiat $2001 ; \quad 70: \quad 105-8$ https://doi.org/10.1136/jnnp.70.1.105

PMCid:PMC1763465

5. Cantu $\mathrm{C}$, Barinagarrementeria $\mathrm{F}$. Cerebral venous thrombosis associated with pregnancy and puerperium: review of 67 cases. Stroke 1993; 24: $1880-4$

https://doi.org/10.1161/01.STR.24.12.1880

PMid:8248971

6. Brain Imaging in Venous Sinus Thrombosis: Practice Essentials, Computed Tomography, Magnetic Resonance Imaging [Internet]. Emedicine.medscape.com. 2018 [cited 18 December 2018]. https://emedicine.medscape.com

7. Poon C, Chang J, Swarnkar A, Johnson M, Wasenko J. Radiologic Diagnosis of Cerebral Venous Thrombosis: Pictorial Review. American Journal of Roentgenology. 2007;189(6_supplement):S64-S75.

8. Masuhr F, Hehraein S, Einhaupl K. Cerebral venous sinus thrombosis. J Neural 2004; 204: 11-23 https://doi.org/10.1007/s00415-004-0321-7

PMid:14999484 